\title{
Investigation of the mechanism of binding of thiacloprid to human serum albumin using spectroscopic techniques and molecular modeling methods
}

\author{
Chuanxian Wang ${ }^{\mathrm{a}}$, Qinghua $\mathrm{Chu}^{\mathrm{a}}$, Changyun Chen $^{\mathrm{b}, *}$ and Zhao Bo ${ }^{\mathrm{c}}$ \\ ${ }^{a}$ Shanghai Exit-Entry Inspection and Quarantine Bureau, Shanghai, China \\ ${ }^{\mathrm{b}}$ School of Biochemical and Environmental Engineering, Nanjing Xiaozhuang University, Nanjing, \\ China \\ ${ }^{\mathrm{c}}$ School of Chemical and Material Science, Nanjing Normal University, Nanjing, China
}

\begin{abstract}
Fluorescence spectroscopy, UV absorption, circular dichroism (CD) spectroscopy and molecular modeling methods were used to characterize the binding properties of thiacloprid (TL) with human serum albumin (HSA) at molecular level under physiological conditions. The fluorescence intensity of HSA decreased regularly with the gradually increasing concentration of thiacloprid. The binding constant $\mathrm{K}$ at three different temperatures (290, 300 and $310 \mathrm{~K}$ ) were $3.07,2.74$ and $1.35 \times 10^{4} \mathrm{M}^{-1}$, respectively, for TL-HSA interaction have been calculated from the relevant fluorescence data. CD spectroscopic measurements have shown that the secondary structures of the protein have been changed by the interaction of thiacloprid with HSA. Furthermore, the study of molecular modeling indicated that thiacloprid could be located on the surface of the binding pocket of subdomains IIA in HSA. The hydrophobic interaction was the major acting force and there are H-bonds and electrostatic interactions between TL and HSA, which is in good agreement with the results from the experimental thermodynamic parameters (the enthalpy change $\Delta H^{0}$ and the entropy change $\Delta S^{0}$ were calculated to be $-20.378 \mathrm{~kJ} / \mathrm{mol}$ and $16.328 \mathrm{~J} / \mathrm{mol} \mathrm{K}$ according to the Van't Hoff equation).
\end{abstract}

Keywords: Binding mechanism, human serum albumin, thiacloprid, spectroscopic techniques, molecular modeling

\section{Introduction}

Chemicals released from agriculture or industry may potentially develop toxic effects in the environment and ecological systems. Among them, pesticides are actively applied and globally used for crop control and to prevent damage to plants, animals, humans or aliments. Thiacloprid (structure shown in Fig. 1), a relatively new neonicotinoid insecticide, ((Z)-3-(6-chloro-3-pyridylmethyl)-1,3-thiazolidin-2ylidenecyanamide) has been produced by Bayer Crop Science since 2003. It acts as an agonist on the nicotinoid acetylcholine receptor (nAChR). Neonicotinoids are systemic insecticides and enter the body via contact and ingestion. They act on the central nervous system of the insects and are mainly used in pest management against biting and sucking insects. At physiological $\mathrm{pH}$ levels, neonicotinoids are not protonated. As a result of this, they have little affinity to the nAChR of vertebrates. Therefore, they

\footnotetext{
* Corresponding author: Changyun Chen, School of Biochemical and Environmental Engineering, Nanjing Xiaozhuang University, Nanjing 211171, China. E-mail: yhjiangccy@126.com.
} 
generally show low acute and chronic toxicity to mammals, birds and fish. However, among neonicotinoids investigated so far, thiacloprid was found to exhibit a comparatively high level of acute toxicity to fish [14].

Human serum albumins have been one of the most studied proteins for many years. They are the most abundant of proteins in blood plasma, accounting for about $60 \%$ of the total protein corresponding to a concentration of $42 \mathrm{~g} / \mathrm{l}$ and provide about $80 \%$ of the osmotic pressure of blood [1,3]. Albumins have been used as a model protein for many and diverse biophysical and physicochemical studies. They play an important role in the transport and deposition of a variety of endogenous and exogenous substances in blood [21]. The globular protein consists of a single polypeptide chain of 585 amino acid residues and has many important physiological functions [2]. The globular protein is composed of three structurally similar domains (I, II and III), each containing two subdomains (A and B) $[5,15]$.

Since the wide spread use of thiacloprid has led to potential toxicological risk to public health, it is necessary to investigate the interaction of thiacloprid with protein, as it can illustrate the nature of TLprotein complex in vitro and provide important insight into the interaction of the physiologically important protein HSA with thiacloprid, which has led to potential toxicological risk to public health [11]. However, so far, none of the investigations were done by determining the TL-HSA binding constants and the effects of thiacloprid complexation on the protein structure in detail. For a better understanding, the study of interaction of thiacloprid with HSA is much needed. Parameters such as mode of interaction, binding constant and number of binding sites are important, these investigations may provide some important theoretic information for the improvement of the metabolism and distribution of thiacloprid in life science, biologic technology, medicine, molecular functional design. To characterize these interactions on the molecular level, optical techniques have become valuable tool because these methods are sensitive and relatively easy to use. As an assistant method, molecular modeling was also employed to explain the binding of thiacloprid to HSA. The results of molecular modeling and the thermodynamic parameters obtained suggest that thiacloprid binds to the hydrophobic cavity of human serum albumin and the hydrophobic interaction is the predominant intermolecular force stabilizing the complex. This study provides a molecular basis for elucidating the mechanism of pesticide acting and predicting unfavorable pesticide interaction. It also provides some basic information of the effect on the function of proteins which reacted with thiacloprid.

\section{Materials and methods}

\subsection{Materials}

Human serum albumin (HSA) was purchased from Sigma Chemical Company. It was used without further purification and its molecular weight was assumed to be 66,500. HSA $(1 \mu \mathrm{M})$ solutions were prepared in ( $\mathrm{pH}$ 7.4) Tris- $\mathrm{HCl}$ buffer solution, and HSA stock solutions were kept in the dark at $277 \mathrm{~K}$. Thiacloprid (grade) was obtained from the National Institute for Control of Pharmaceutical and Bioproducts, China. The stock solution was prepared in methanol because thiacloprid easily dissolve in the methanol. The concentration of thiacloprid is $5 \times 10^{-3} \mathrm{~mol} / \mathrm{l}$. All the stock solutions were then diluted with buffer solution to obtain the actual assay solutions. $\mathrm{NaCl}$ (analytical grade, $1.0 \mathrm{~mol} / \mathrm{l}$ ) solution was used to maintain the ion strength at 0.1 . The buffer $(\mathrm{pH} 7.4)$ consists of Tris $(0.2 \mathrm{~mol} / \mathrm{l})$ and $\mathrm{HCl}$ $(0.1 \mathrm{~mol} / \mathrm{l})$. The $\mathrm{pH}$ was checked with a suitably standardized $\mathrm{pH}$ meter (pHS-3C). The thiacloprid was titrated by micro-injector. All reagents were of analytical reagent grade and distilled water was used throughout the experiments. 


\subsection{Apparatus and methods}

\subsubsection{UV-absorbance titration}

UV absorption spectra were recorded on an UV-2450 spectrophotometer. Quartz cuvettes with a path length of $1 \mathrm{~cm}$ were used. The absorbance titration were performed by keeping the concentration of HSA $(1.0 \mu \mathrm{M})$ constant while varying the concentration of thiacloprid $(0-25.0 \mu \mathrm{M})$. An equal amount of thiacloprid was titrated into the reference cell at the same time.

\subsubsection{Fluorescence titration}

Fluorescence spectra was recorded on a LS 50B spectrofluorimeter (Perkin-Elmer, USA). A slit width of $5 \mathrm{~nm} / 5 \mathrm{~nm}$ was typically used $3.0 \mathrm{ml}$ solution containing appropriate concentration of HSA $(1.0 \mu \mathrm{M})$ was titrated manually by successive additions of $5 \times 10^{-3} \mathrm{M}$ solution of thiacloprid (to give a final concentration of $0-35 \mu \mathrm{M})$. The total content of titration was not more than $3.5 \%$ of the volume of the protein. The $\mathrm{pH}$ measurements were carried out on a PHS-3C Exact Digital pH meter. Protein mixed with the thiacloprid was maintained the full $10 \mathrm{~min}$ before the measurement for the sufficient reaction. The emission spectra were obtained in the rang from 300 to $500 \mathrm{~nm}$, with the excitation wavelength at $280 \mathrm{~nm}$ and the emission at $352 \mathrm{~nm}$. All experiments were measured at different temperature 290, 300 and $310 \mathrm{~K}$, respectively.

\subsubsection{Circular dichroism spectroscopy}

Circular dichroism were performed on a Jasco-20 automatic recording spectropolarimeter (Japan), using a $2-\mathrm{mm}$ quartz cell at room temperature, and the speed of scanning was $20 \mathrm{~nm} / \mathrm{min}$. The spectra were recorded in the far-UV region which range from $200-250 \mathrm{~nm}$. The protein concentration used in our experiment was $1.0 \times 10^{-6} \mathrm{M}$. And the buffer solution was selected as the blank and was automatically subtracted during scanning.

\subsubsection{Molecular modeling}

Protein-ligand docking study was performed using Discovery Studio 2.1 software where the $\mathrm{pH}$ is set to 7.4. The crystal structure of HSA (PDB entry code 1h9z) was downloaded from the Protein Data Bank [16] and the potential of the 3D structure of HSA was assigned to the CHARMm force field. The structure of thiacloprid was built in Discovery Studio 2.1 and optimized by applying the CHARMm force field. During the docking process, a maximum of 10 conformers have been chosen for the final energy minimization. The conformation with the lowest energy was selected for final analysis.

\section{Results and discussion}

\subsection{UV-absorbance spectra of HSA with thiacloprid}

UV/vis absorption measurement is a simple and effective method in detecting complex formation. The UV absorption spectrum of HSA in the absence and presence of thiacloprid are shown in Fig. 1. The complex of TL-HSA is evident from UV-vis absorption spectral data. HSA has strong absorbance with a peak at $204 \mathrm{~nm}$ and the absorbance of HSA decrease with the addition of thiacloprid. The peak position of HSA has a little red shift. The results indicated that an interaction occurred between thiacloprid and HSA. In addition, the absorbance values $(280 \mathrm{~nm})$ of the system are very low $(<0.05)$ as in Fig. 1A. Therefore, inner filter effects and re-absorption were negligible [12]. This result indicates that the probable quenching mechanism is due to the formation of the complex between thiacloprid and HSA. 


\subsection{Analysis of fluorescence quenching of HSA by thiacloprid}

Fluorescence measurements were carried out to investigate whether thiacloprid interacts with HSA. Figure 2 shows the fluorescence emission spectra of HSA in the absence and presence of thiacloprid. HSA has a strong fluorescence emission peak at $352 \mathrm{~nm}$ on excitation at $280 \mathrm{~nm}$. Gradual addition of thiacloprid to the HSA solution leads to a small bathochromic shift of the emission maximum accompany by an decrease of fluorescence intensity. The observations reflect that the microenvironment around the fluorophore in the protein solution is quite different from that of pure aqueous phase. The changes in the fluorometric behavior of the fluorophore with the addition of thiacloprid in HSA solution can be rationalized in terms of binding of the pesticide with the protein leading to a more polar microenvironment around the fluorophore. A red shift in the fluorescence maximum also suggests an enhancement in the polarity of the microenvironment.

The static and dynamic quenching is distinguished by the results at different temperatures [4]. Higher temperature results in faster diffusion and larger amounts of collisional quenching and will typically

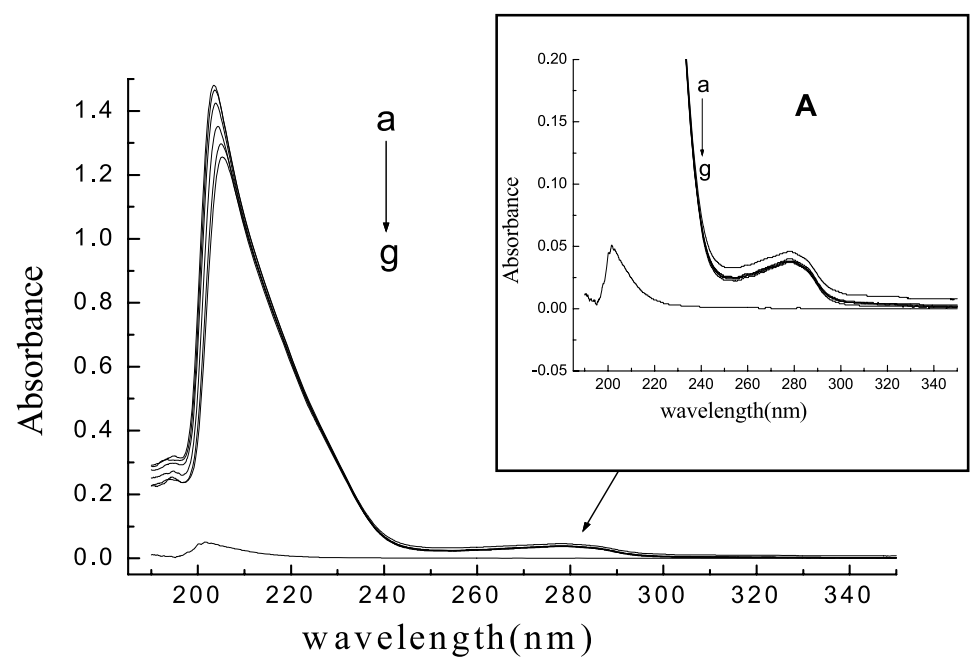

Fig. 1. UV absorption spectrum obtained in Tris- $\mathrm{HCl}$ buffer solution ( $\mathrm{pH} 7.4)$ : (a) the absorption spectrum of HSA, $\mathrm{C}_{\mathrm{HSA}}=1.0 \mu \mathrm{M}$; (b)-(f) TL-HSA, $1 \mu \mathrm{M}$ HSA in the presence of 1.0, 2.0, 4.0, 7.0, 8.0 $\mu \mathrm{M} \mathrm{TL},(\mathrm{g})$ the absorption spectrum of $1.0 \mu \mathrm{M}$ TL.

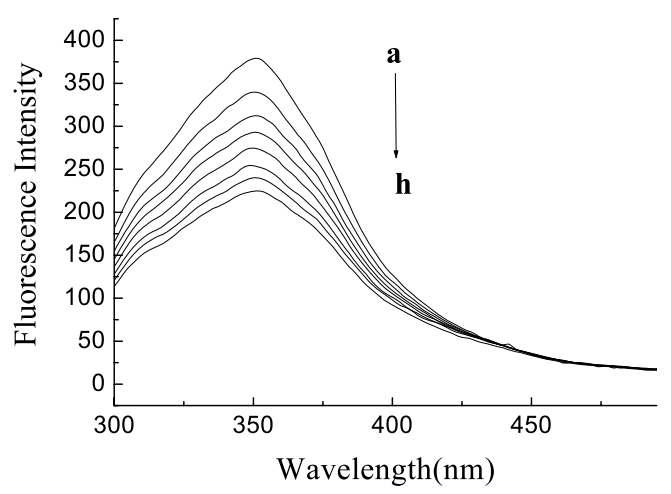

Fig. 2. Fluorescence emission spectra at $\mathrm{pH} 7.4$ : (a) $1.0 \mu \mathrm{M}$ HSA; (b)-(h), respectively, $1.0 \mu \mathrm{M}$ HSA in the presence of 5.0, 10, $15,20,25,30,35 \mu \mathrm{M}$ TL. 


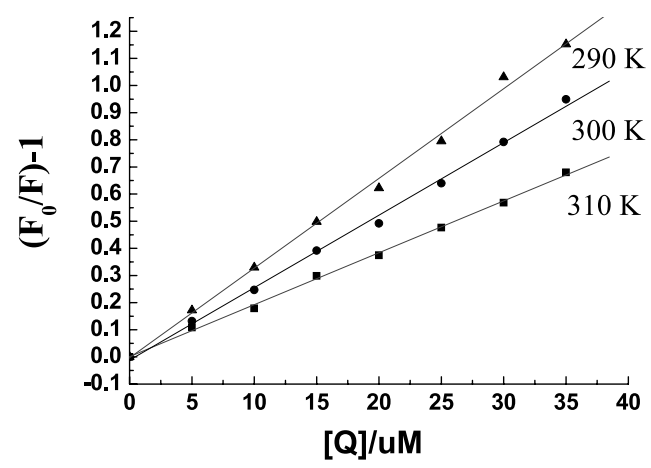

Fig. 3. The Stern-Volmer curves for the binding of TL with HSA at different temperatures. Plot of $F_{0} / F-1$ against $[Q]$ for

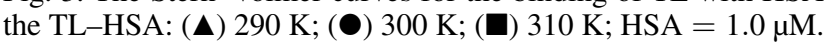

leads to the dissociation of weakly bound complexes. Therefore, the quenching constant increases with increasing temperature for dynamic quenching. However, it decreases with increase in temperature for static quenching. The possible quenching mechanism can be interpreted by fluorescence quenching spectra of the protein and the $F_{0} / F-1-[Q]$ (Stern-Volmer) curves of HSA with thiacloprid at different temperatures 290, 300 and $310 \mathrm{~K}$ as shown in Fig. 3. It can be found from Fig. 3 that the Stern-Volmer plots were linear and the slopes decreased with increasing temperature, which is consistent with the static type of quenching mechanism. According to Eq. (1):

$$
F_{0} / F=1+K_{\mathrm{q}} \tau_{0}[Q]=1+K_{\mathrm{SV}}[Q]
$$

where $F_{0}$ and $F$ are the fluorescence intensities of HSA in absence and presence of quencher, respectively, $K_{\mathrm{SV}}$ is the Stern-Volmer quenching constant and $[Q]$ is the concentration of quencher (thiacloprid). Quenching constant $K_{\mathrm{SV}}$ can be calculated from Fig. 3 and their values are $3.304 \times 10^{4}$, $2.672 \times 10^{4}, 1.911 \times 10^{4} \mathrm{l} / \mathrm{mol}$ at 290,300 and $310 \mathrm{~K}$, respectively. These results indicate that the probable quenching mechanism of fluorescence of HSA by thiacloprid is a static quenching process and can predict that a complex formed between thiacloprid and HSA. The interaction was decreased when the temperature rose, thus the quenching efficiency of thiacloprid to HSA was reduced when the experimental temperature rose.

\subsection{Determinations of binding constants and the number of binding site}

For static quenching, the relationship between fluorescence quenching intensity and the concentration of quenchers can be described by the binding constant formula [23]:

$$
\log \left(F_{0}-F\right) / F=\log K_{\mathrm{b}}+n \log [Q],
$$

where $K_{\mathrm{b}}$ is the binding constant and $n$ is the number of binding sites. After the fluorescence quenching intensities were measured, the double-logarithm algorithm was assessed by Eq. (2). The binding constants $\left(K_{\mathrm{b}}\right)$ and the number of binding sites $(n)$ presents in Table 1 . The result illustrates that there is a strong binding force between thiacloprid and HSA, and the number of binding sites is independent of temperature from 290 to $310 \mathrm{~K}$. The obtained value $n=0.988 \pm 0.02$ indicates that almost one molecule of thiacloprid binds to a HSA molecule and it corresponds to the high affinity binding site. It also 
shows that the values of $K_{\mathrm{b}}$ decrease with the rising temperature, which is in good agreement with the trend of $K_{\mathrm{SV}}$ as mentioned above. It implies that an unstable complex may be formed in the binding reaction and the complex would possibly be dissociated partly when the temperature increases.

\subsection{Thermodynamic analysis and determination of the molecular forces}

The molecular forces between a small molecular substance and macromolecule mainly include hydrogen bond, van der Waals force, electrostatic force, hydrophobic interaction force and so on. Considering the dependence of binding constant on temperature, a thermodynamic process was deemed to be responsible for the formation of the complex. On this purpose, the temperature dependence of the binding constant was studied. In this section, the association constants obtained by the Stern-Volmer was used to calculate the thermodynamic parameters. Thermodynamic parameters were calculated based on the temperature dependence of the quenching constant for TL-HSA binding.

The temperatures chosen were 290,300 and $310 \mathrm{~K}$. By plotting the quenching constants according to Van't Hoff equation, the thermodynamic parameters were determined from a linear Van't Hoff plot and listed in Table 2. It is clear from the values of standard entropy changes $\left(\Delta S^{0}\right)$ and standard enthalpy changes $\left(\Delta H^{0}\right)$ that the binding of thiacloprid to HSA is an exothermic process accompanied by a positive value of $\Delta S^{0}$ and a negative value of $\Delta G^{0}$. The binding process is always spontaneous as evidenced by the negative sign of $\Delta G^{0}$ value. For pesticide-protein interaction, Ross and Subramanian [17] have characterized the sign and magnitude of the thermodynamic parameters associated with these various kinds of interactions that play an important role in the protein association process. From the point of the view, a positive $\Delta H^{0}$ and $\Delta S^{0}$ value is associated with hydrogen bonding interaction. The negative $\Delta H^{0}$ and $\Delta S^{0}$ values are associated with hydrogen bonding and van der Waals interaction in low dielectric medium. Specific electrostatic interaction between ionic species in an aqueous solution is characterized by positive $\Delta S^{0}$ value and very small negative $\Delta H^{0}$ value, almost zero [18]. The negative $\Delta H^{0}$ and positive $\Delta S^{0}$ values are associated with hydrophobic force and electrostatic force interaction. Thus it is difficult to interpret the thermodynamic parameters of TL-HSA interaction with a single intermolecular force. The interaction force between small molecules and proteins is not only a single force in general. May be there are variety of forces existing in the interaction forces, which contains the electrostatic force. It is believed that the hydrophobic small pharmacological molecule cuttage grafting to protein molecules within the hydrophobic region, when a small pharmacological molecule combine with proteins. The solubility of thiacloprid in water is very small, so thiacloprid is belonging to hydrophobic

Table 1

The binding constant $K_{\mathrm{b}}$ and the number of binding site $n$ at different temperatures

\begin{tabular}{llccc}
\hline$T(\mathrm{~K})$ & Linear equation & $R$ & $10^{4} K_{\mathrm{A}}\left(\mathrm{M}^{-1}\right)$ & $n$ \\
\hline 290 & $\log \left[\left(F_{0}-F\right) / F\right]=4.4869+0.9936 \log [Q]$ & 0.9999 & 3.07 & 0.9936 \\
300 & $\log \left[\left(F_{0}-F\right) / F\right]=4.4374+1.0036 \log [Q]$ & 0.9997 & 2.74 & 1.0036 \\
310 & $\log \left[\left(F_{0}-F\right) / F\right]=4.1289+0.9667 \log [Q]$ & 0.9998 & 1.35 & 0.9667 \\
\hline
\end{tabular}

Table 2

Thermodynamic parameters of TL-HSA

\begin{tabular}{lccc}
\hline$T(\mathrm{~K})$ & $\Delta G^{0}(\mathrm{~kJ} / \mathrm{mol})$ & $\Delta S^{0}(\mathrm{~J} /(\mathrm{mol} \cdot \mathrm{K}))$ & $\Delta H^{0}(\mathrm{~kJ} / \mathrm{mol})$ \\
\hline 290 & -25.113 & & \\
300 & -25.276 & 16.328 & -20.378 \\
310 & -25.439 & & \\
\hline
\end{tabular}


small molecule. The drug molecule embedded in the internal hydrophobic region of protein molecules may be responsible for the fluorescence quenching $[8,13]$. Therefore, the binding of thiacloprid to HSA might involve hydrophobic interaction strongly as evidenced by the positive value of $\Delta S^{0}$ although the role of electrostatic interaction cannot be excluded. From molecular modeling we found that hydrogen bonding force also plays a major role.

\subsection{Changes of the HSA secondary structure induced by thiacloprid binding from $C D$}

To obtain more information on the binding of thiacloprid to HSA, CD spectroscopy was used to study the structure of HSA and TL-HSA complex. CD, a sensitive technique to monitor the conformational change in the protein. The CD spectra of HSA and TL-HSA complex were shown in Fig. 4. As can be seen from Fig. 4, the CD spectra of HSA exhibits two negative bands in the ultraviolet region at 208 and $223 \mathrm{~nm}$, which is a characteristic of $\alpha$-helical structure of protein. The reasonable explanation is that the negative peaks between 208-209 and 222-223 nm are both contributed by $n \rightarrow \pi^{*}$ transfer for the peptide bond of $\alpha$-helical [22]. The addition of thiacloprid to HSA leads to a decrease in the ellipticity without significant shift of the peaks, indicating that the binding of thiacloprid to HSA induces a decrease in the $\alpha$-helical content of HSA. However, the CD spectra of HSA in the presence and absence of thiacloprid were similar in shape, which means the structure of HSA was also predominated by $\alpha$-helix after the binding of thiacloprid [20]. The binding of thiacloprid to HSA causes an decrease with negative Cotton effect in band intensity, indicating the considerable changes in the protein secondary structure with the decrease of $\alpha$-helical structure, and it may be the result of the formation of TL-HSA complex. The CD result was expressed as MRE (Mean Residue Ellipticity) in $\mathrm{deg} \cdot \mathrm{cm}^{2} \cdot \mathrm{dmol}^{-1}$, which is defined as:

$$
\mathrm{MRE}=\theta_{\mathrm{obs}} /(10 C p n l),
$$

where $\theta_{\text {obs }}$ is the CD in millidegree, $n$ is the number of amino acid residues (585), $l$ is the path length of the cell $(1 \mathrm{~cm})$ and $C p$ is the molar concentration of HSA. The $\alpha$-helical contents of free and combined HSA were calculated from the MRE values at $208 \mathrm{~nm}$ using the equation [7]:

$$
\alpha \text {-helix }(\%)=\left\{\left(-\mathrm{MRE}_{208}-4000\right) /(33,000-4000)\right\} \times 100 \text {. }
$$

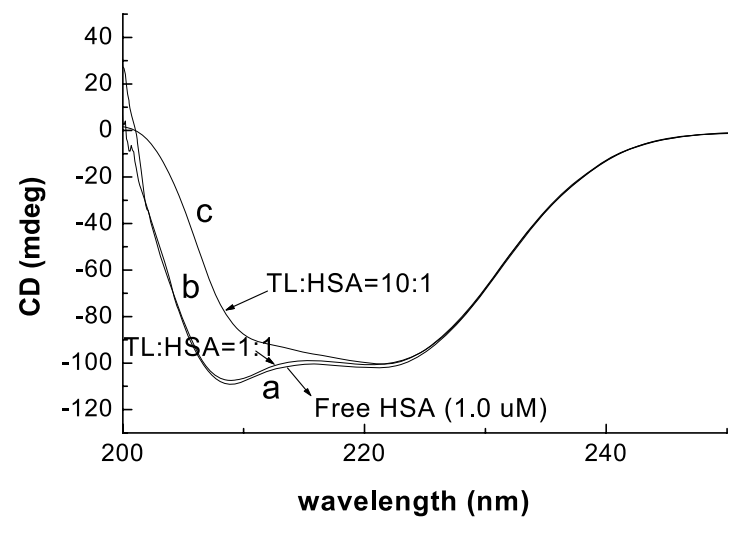

Fig. 4. CD spectra of the HSA-TL system. (a) $1.0 \times 10^{-6} \mathrm{~mol} / \mathrm{l} \mathrm{HSA}$; (b) $1.0 \times 10^{-6} \mathrm{~mol} / \mathrm{l} \mathrm{HSA}$ in the presence of $1.0 \times 10^{-6} \mathrm{~mol} / 1 \mathrm{TL}$; (c) $1.0 \times 10^{-6} \mathrm{~mol} / \mathrm{l} \mathrm{HSA}$ in the presence of $1.0 \times 10^{-5} \mathrm{~mol} / \mathrm{l} \mathrm{TL}$. 
The calculated results exhibit an decrease of $\alpha$-helical structure from 49.69 to $48.88 \%$ at molar ratio of TL/HSA of 1:1 and from 49.69 to $29.86 \%$ at molar ratio of TL/HSA of 10:1. The results reveal that the binding of thiacloprid to HSA has changed the secondary structure of HSA. The percentage $\alpha$-helix structure of HSA decreased indicated that thiacloprid bound with the amino acid residue of the main polypeptide chain of HSA and destroyed their hydrogen bonding networks [9].

\subsection{Molecular modeling of the complex of HSA and thiacloprid}

The application of molecular modeling has been employed to study the interaction between TL and HSA. Crystal structure analysis has revealed that human serum albumin comprises of three homologous domains (I-III): I (residues 1-195), II (residues 196-383), III (residues 384-585) and each containing two subdomains (A and B). The principal [19] regions of ligand binding to HSA are located in hydrophobic cavities in subdomains IIA and IIIA, which are consistent with sites I and II, respectively, and the sole tryptophan residue (Trp-214) is in subdomain IIA of HSA [6].

The stereoview of the docking pose of TL with HSA is shown in Fig. 5. As shown in Fig. 5, TL is best located on the surface of the binding pocket of subdomain IIA. The binding free energy $(\Delta G)$ was found to be $-21.34 \mathrm{~kJ} / \mathrm{mol}$. Negative value of $\Delta G$ shows that the binding reaction is thermodynamically favorable.

The amino acid residues involved in the binding of TL to HSA were predicted in Fig. 6 where only residues around $5 \AA$ of TL were displayed. The pyridine ring of TL is making hydrophobic interactions

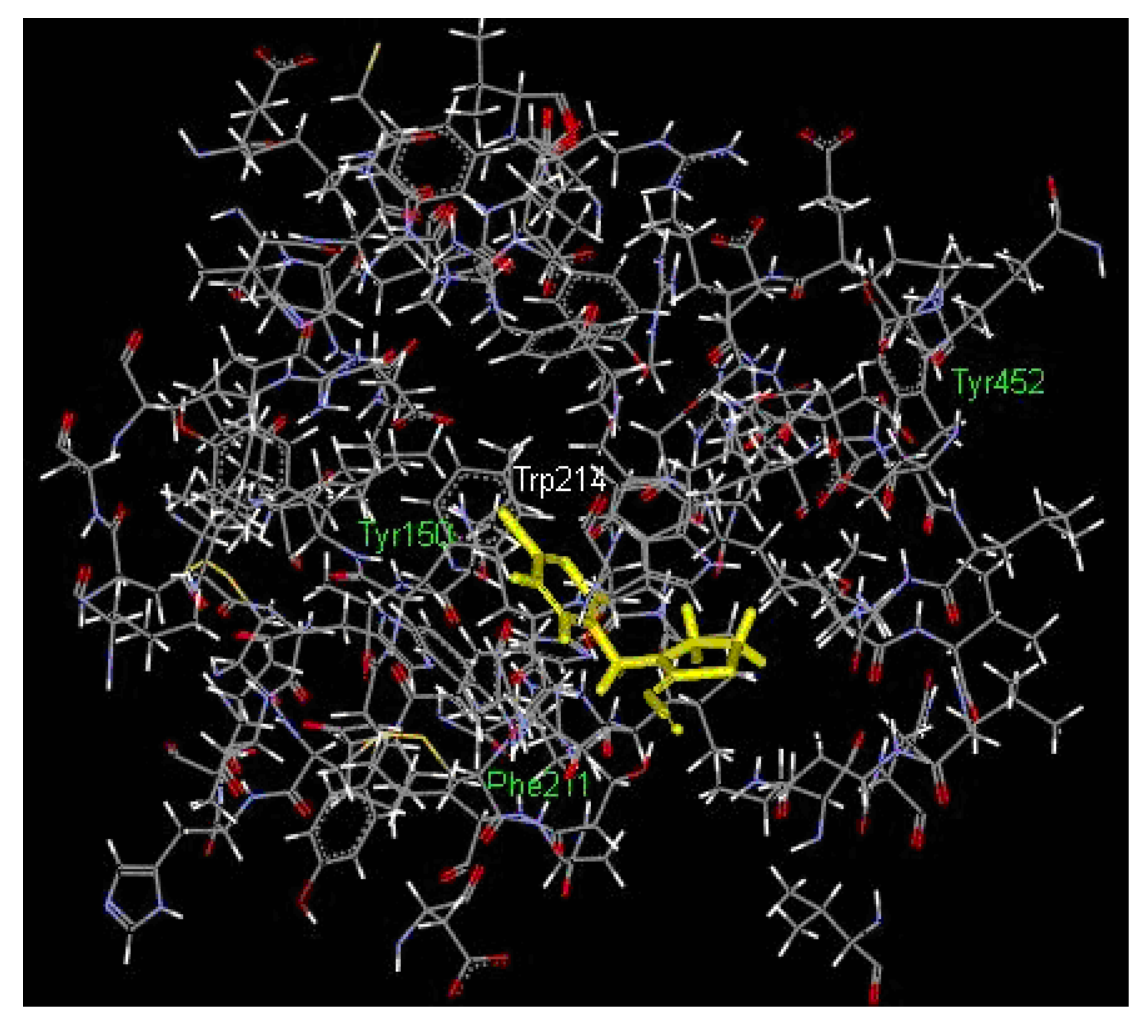

Fig. 5. The stereoview of the docking pose of TL with HSA. (Colors are visible in the online version of the article; http://dx.doi. org/10.3233/SPE-2011-0498.) 


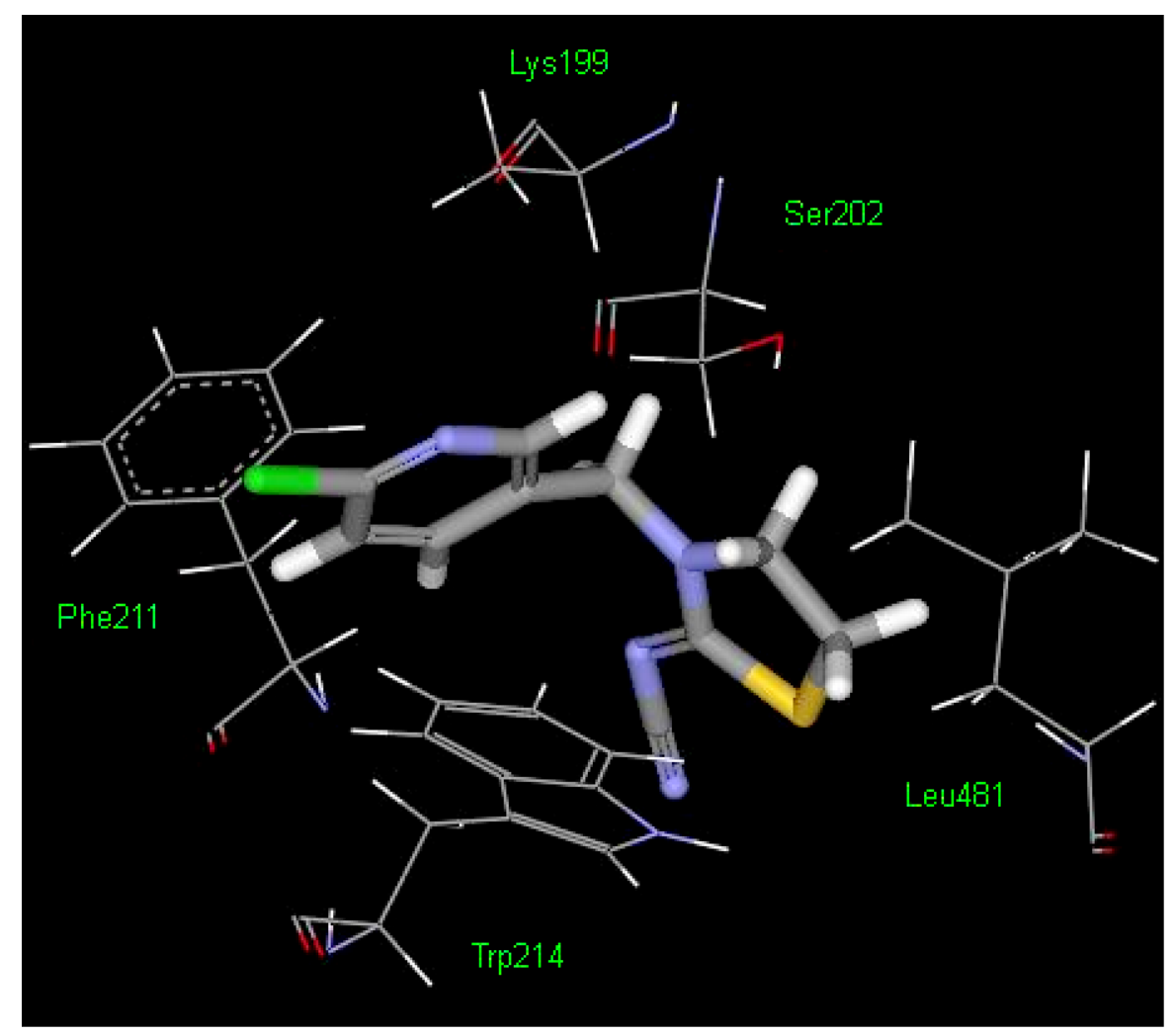

Fig. 6. The amino acid residues involved in the binding of TL to HSA. (Colors are visible in the online version of the article; http://dx.doi.org/10.3233/SPE-2011-0498.)

with Phe211, Trp214. The interaction between thiacloprid and HSA is not exclusively hydrophobic as there are several ionic and polar residues (Lys199, Ser202 and Leu481) in the proximity of bound ligand (within $5 \AA$ A). These polar residues play an inportant role in stabilizing drag via $\mathrm{H}$-bonds and electrostatic interactions. Trp214 is in a suitable position involved in making H-bonds with the imino groups of the side chain. The hydrogen-bonding or electrostatic interaction acts as an anchor and this helps to attain the 3D space position of drug in its binding pocket. This facilitates the hydrophobic interaction of the pyridine ring with the side chain of HSA residues.

\section{Conclusions}

The results of fluorescence quenching measurements and molecular modeling study suggested that thiacloprid could bind to HSA through the hydrophobic interaction and hydrogen bonding with high affinity. The probable binding domain of thiacloprid was at the hydrophobic pocket located in subdomain IIA. The spectroscopic evidence based on CD spectroscopy also showed that the secondary structure of HSA changed after thiacloprid bound to HSA. The binding constant and the standard free energy change between the thiacloprid and HSA was obtained based on the fluorescence results. The results indicated that the interaction is a static quenching process. The binding study of thiacloprid with proteins has toxicological importance. This study is expected to provide important insight into the interactions of the physiologically important protein HSA with thiacloprid congeners. 


\section{Acknowledgements}

This work was supported by the Nanometer Technology Special Project (No. 1052NM06700), and the Technology Standard Project (No. 08DZ0505000) of Shanghai Municipal Science and Technology Commission, and High-tech Industrialization Project (JHZD09-18) of Jiangsu Education Department.

\section{References}

[1] J.R. Brown and P. Shockley, Serum albumin: structure and characterization of its ligand binding sites, in: Lipid Protein Interactions, Vol. 1, P.C. Jost and O.H. Griath, eds, Wiley, New York, 1982, pp. $26-68$.

[2] D.C. Carter, X.M. He, S.H. Munson, P.D. Twigg, K.M. Gernert, M.B. Broom and T.Y. Miller, Three-dimensional structure of human serum albumin, Am. Assoc. Adv. Sci. 244(4909) (1989), 1195-1198.

[3] D.C. Carter and J.X. Ho, Structure of serum albumin, Adv. Protein Chem. 2(45) (1994), 153-203.

[4] G.Z. Chen, X.Z. Huang, J.G. Xu, Z.Z. Zheng and Z.B. Wang, The Methods of Fluorescence Analysis, 2nd edn, Science Press, Beijing, 1990, p. 50.

[5] S. Curry, P. Brick and N.P. Frank, Atomic structure and chemistry of human serum albumin, Biochem. Biophys. Acta 13(1441) (1999), 269-272.

[6] S. Curry, H. Mandelkow, P. Brick and N. Franks, Crystal structure of human serum albumin complexed with fatty acid reveals an asymmetric distribution of binding sites, Nat. Struct. Biol. 5 (1998), 827-835.

[7] H. Gao, Y.N. Wang, Y.G. Fan and J.B. Ma, Interactions of some modified mono- and bis- $\beta$-cyclodextrins with bovine serum albumin, Bioorg. Med. Chem. 14 (2006), 131-137.

[8] B. Huang, G.L. Zou and Y.M. Yang, Studies on the interaction between adriamy and bovine serun albumin, Acta Chim. Sinica 60(10) (2002), 1867-1871.

[9] J. Kang, Y. Liu, M.-X. Xie, S. Li, M. Jiang and Y.D. Wang, Interactions of human serum albumin with chlorogenic acid and ferulic acid, Biochim. Biophys. Acta 1674 (2004), 205-214.

[10] J.R. Lakowicz, Principles of Fluorescence Spectroscopy, Plenum Press, New York, 1983, pp. 257-295.

[11] J. Li, X. Liu, C. Ren, J. Li, F. Sheng and Z. Hu, In vitro study on the interaction between thiophanate methyl and human serum albumin, J. Photochem. Photobiol. B 94 (2009), 158-163.

[12] J. Liu, J.-N. Tian, J. Zhuang, Z. Hu and X. Chen, Interaction of magnololl with bovine serum albumin: a fluorescencequenching study, Anal. Bioanal. Chem. 376 (2003), 864-867.

[13] X.F. Liu, Y.M. Xia, Y. Fang et al., Interaction between natural pharmaceutical homologued of coumarin and bovine serum albumin, Acta Chim. Sinica 64(16) (2004), 1484-1490.

[14] R. Osterauer and H.-R. Köhler, Temperature-dependent effects of the pesticides thiacloprid and diazinon on the embryonic development of zebrafish (Danio rerio), Aquat. Toxicol. 86 (2008), 485-494.

[15] T. Peters, Serum albumin, Adv. Protein Chem. 37 (1985), 161-242.

[16] I. Petitpas, A.A. Bhattacharya, S. Twine, M. East and S. Curry, Crystal structure analysis of warfarin binding to human serum albumin: anatomy of drug site I, J. Biol. Chem. 276(25) (2001), 22804-22809.

[17] P.D. Ross and S. Subramanian, Thermodynamics of protein association reactions: forces contributing to stability, Biochemistry 20 (1981), 3096-3102.

[18] I. Sjoholm, B. Ekman, A. Kober, I. Ljungstedt-Pahlman, B. Seiving and T. Sjodin, Binding of drugs to human serum albumin: XI. The specificity of three binding sites as studied with albumin immobilized in microparticles, Mol. Pharmacol. 16(3) (1979), 767-777.

[19] G. Sudlow, D.J. Birkett and D.N. Wade, Further characterization of specific drug binding site on human serum albumin, Mol. Pharmacol. 12 (1976), 1052-1061.

[20] S. Sugio, A. Kashima, S. Mochizuki, M. Noda and K. Kobayashi, Crystal structure of human serum albumin at $2.5 \AA$ resolution, Protein Eng. 12(6) (1999), 439-446.

[21] K. Yamasaki, T. Maruyama, U. Kragh-Hansen and M. Otagiri, Characterization of site I on human serum albumin: concept about the structure of a drug binding site, Biochem. Biophys. Acta 1295 (1996), 57-147.

[22] P. Yang and F. Gao, The Principle of Bioinorganic Chemistry, Science Press, Beijing, 2002, pp. 322-342.

[23] Q.J. Zhou, J.F. Xiang, Y.L. Tang, J.P. Liao, C.Y. Yu, H. Zhang, L. Li, Y.Y. Yang and G.Z. Xu, Investigation on the interaction between a heterocyclic aminal derivative, SBDC, and human serum albumin, Colloids Surf. B 61 (2008), $75-80$. 


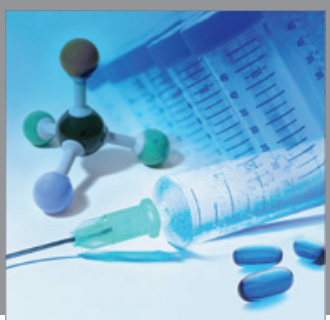

International Journal of

Medicinal Chemistry

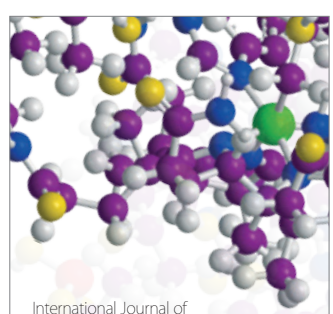

Carbohydrate Chemistry

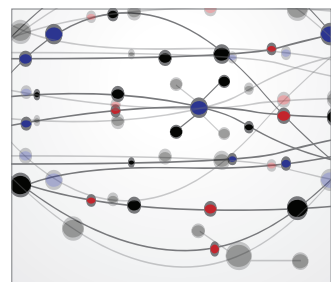

The Scientific World Journal
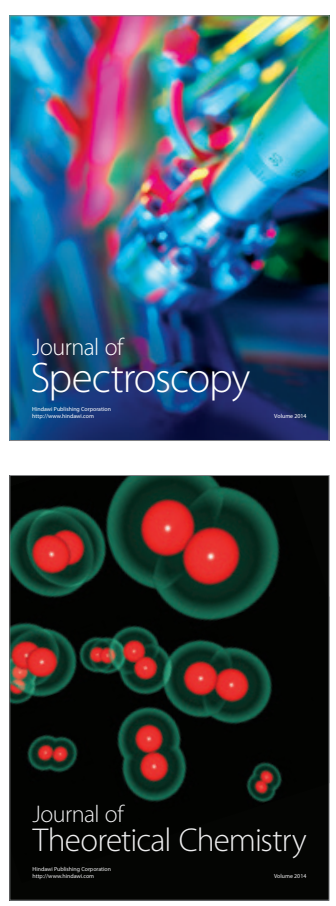
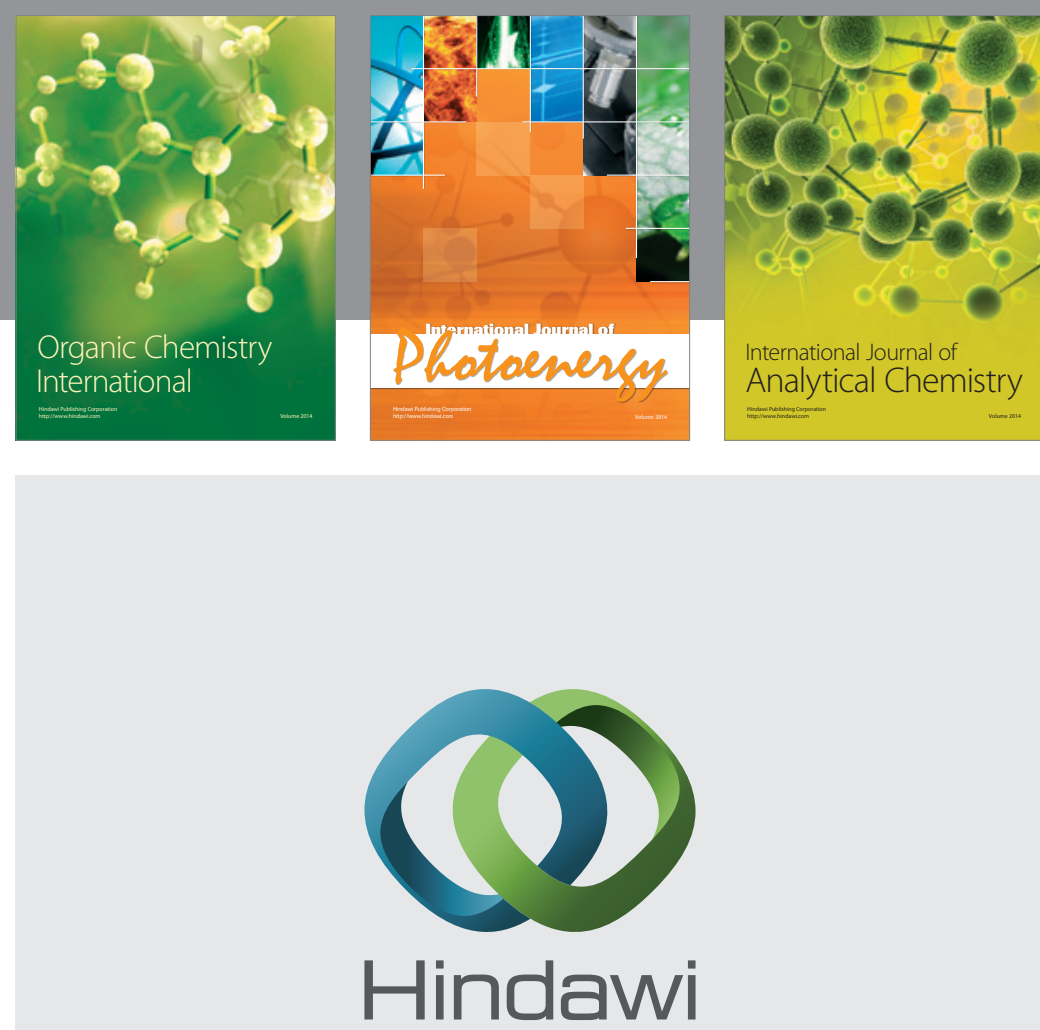

Submit your manuscripts at

http://www.hindawi.com
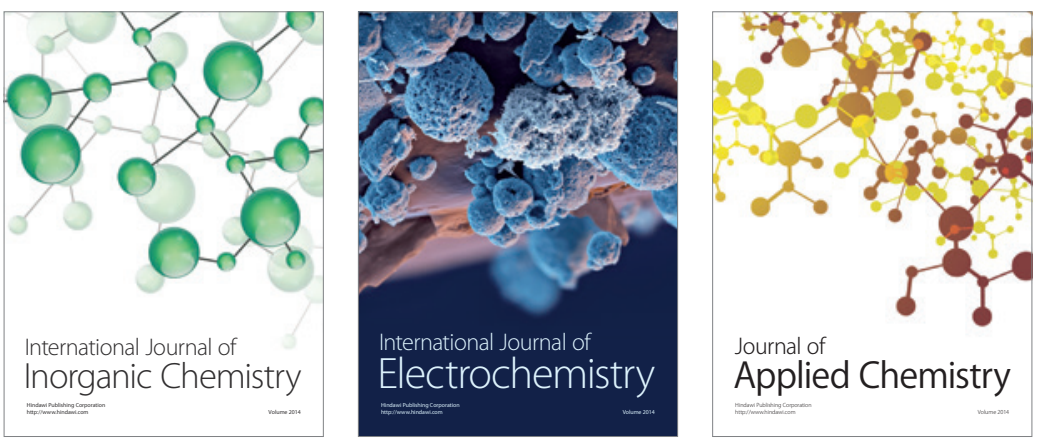

Journal of

Applied Chemistry
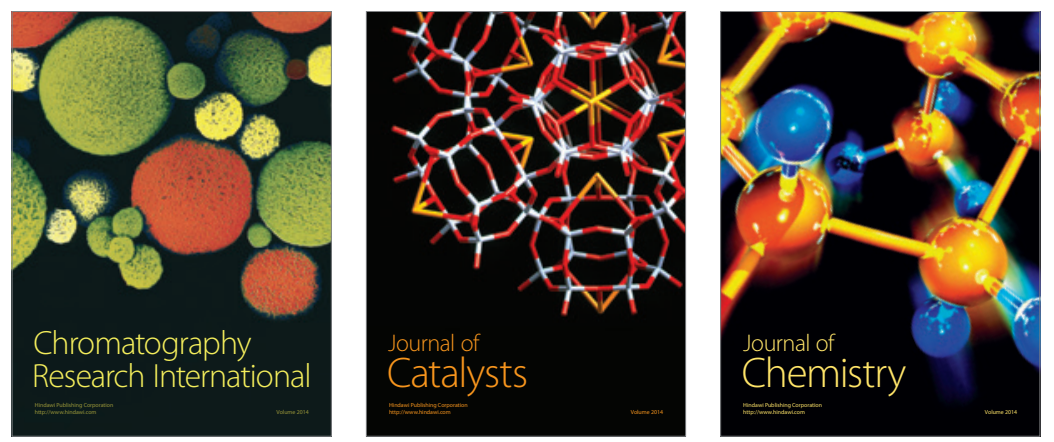
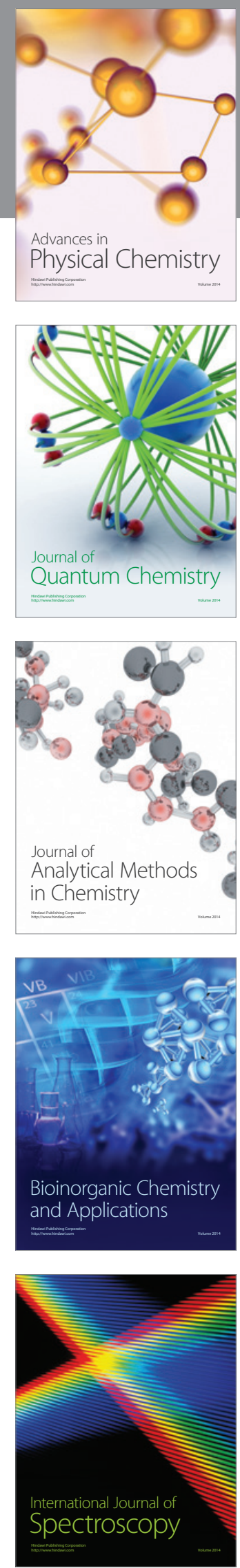